\title{
Mobile-phone-based Rheinberg microscope with a light-emitting diode array
}

Yuma Ogasawara

Ryo Sugimoto

Ryoji Maruyama

Hidenobu Arimoto

Yosuke Tamada

Wataru Watanabe 


\title{
Mobile-phone-based Rheinberg microscope with a light-emitting diode array
}

\author{
Yuma Ogasawara, ${ }^{\mathrm{a}}$ Ryo Sugimoto, ${ }^{\mathrm{a}}$ Ryoji Maruyama, ${ }^{\mathrm{a}}$ Hidenobu Arimoto, ${ }^{\mathrm{b}}$ Yosuke Tamada, ${ }^{\mathrm{c}}$ and \\ Wataru Watanabe ${ }^{a, *}$ \\ ${ }^{a}$ Ritsumeikan University, College of Science and Engineering, Department of Electrical and Electronic Engineering, Kusatsu, Japan \\ ${ }^{b}$ National Institute of Advanced Industrial Science and Technology, Electronics and Photonics Research Institute, Tsukuba, Japan \\ 'National Institute for Basic Biology, Division of Evolutionary Biology, Okazaki, Japan
}

\begin{abstract}
Mobile phone technology has led to implementation of portable and inexpensive microscopes. Lightemitting diode (LED) array microscopes support various multicontrast imaging by flexible illumination patterns of the LED array that can be achieved without changing the optical components of the microscope. Here, we demonstrate a mobile-phone-based LED array microscope to realize multimodal imaging with bright-field, dark-field, differential phase-contrast, and Rheinberg illuminations using as few as 37 LED bulbs. Using this microscope, we obtained high-contrast images of living cells. Furthermore, by changing the color combinations of Rheinberg illumination, we were able to obtain images of living chromatic structures with enhanced or diminished contrast. This technique is expected to be a foundation for high-contrast microscopy used in modern field studies. $\odot$ The Authors. Published by SPIE under a Creative Commons Attribution 4.0 Unported License. Distribution or reproduction of this work in whole or in part requires full attribution of the original publication, including its DOI. [DOI: 10.1117/1.JBO.24.3.031007]

Keywords: bright-field image; dark-field image; differential phase contrast image; smartphone; portable microscope; light-emitting diode array microscopy; Rheinberg illumination.
\end{abstract}

Paper 180381SSR received Jun. 30, 2018; accepted for publication Aug. 27, 2018; published online Sep. 24, 2018.

\section{Introduction}

Molecular biology studies in the field have become active to elucidate the responses of organisms to fluctuating natural environments at molecular and cellular levels. ${ }^{1,2}$ For such field studies and onsite monitoring, compact and cost-effective microscopes are necessary to understand the presampling conditions of living cells, tissues, and organisms at the sites where they grow naturally. To this end, mobile phone technology leads to implementation of portable and inexpensive microscopes with ease of operation. ${ }^{3-9}$ Most mobile-phone-based optical microscopes are useful to observe bright-field images or fluorescence images. Recently, dual-mode optical microscopy using a mobile phone has been reported for acquisition of bright-field and dark-field images with the light-emitting diodes (LEDs) of a mobile phone and ambient light. ${ }^{10}$

The LED array microscope can provide simultaneous acquisition of multicontrast images by changing bright-field, darkfield, and differential phase-contrast (DPC) illumination patterns of the LED array. ${ }^{11-13}$ Philips et al. ${ }^{14}$ demonstrated a multicontrast microscope with an LED array on a smartphone platform. Jung et al. ${ }^{15}$ demonstrated a smartphone-based multicontrast microscope using color-multiplexed illumination. We have reported a smartphone-based multicontrast microscope with different numerical apertures (NAs). ${ }^{16}$ Multicontrast images with bright-field, dark-field, and DPC illuminations are useful for observation of biological cells. Nevertheless, some other methods of contrast enhancement are required. Rheinberg illumination microscopy enables simultaneous acquisition of bright-field and dark-field images by two-color illumination and is originally used for visual enhancement of the contrast between a dark-field image and a bright-field image because the bright-

*Address all correspondence to: Wataru Watanabe, E-mail: wata-w@fc .ritsumei.ac.jp field image becomes the background color. ${ }^{17,18}$ Moreover, simultaneous acquisition of bright-field and dark-field images using microscopy is useful for observing living cells and organisms, which generally show movement. Such images are therefore particularly beneficial when obtained using field-portable microscopes. However, no study of Rheinberg microscopy using field-portable microscopes has been reported in the relevant literature.

As described in this paper, we demonstrate a mobile-phonebased microscope to realize multicontrast imaging with brightfield, dark-field, DPC, and Rheinberg illuminations with as few as 37 LED bulbs. Multimodal images are instantly obtainable by changing the LED array illumination patterns and colors. Additionally, we investigated the color combination of Rheinberg illumination and obtained images of chromatic structures in living cells with enhanced or diminished contrast.

\section{Rheinberg Illumination with an LED Array}

In Rheinberg illumination, a bright-field image and a dark-field image are obtainable by two-color illumination. Figure 1(a) explains an LED array illumination pattern with which a researcher can perform Rheinberg illumination. The central rays of red light from the LED array pass through a sample and serve as bright-field illumination. The oblique green rays from outer ring LEDs hit the sample and serve as dark-field illumination. In general dark-field imaging, the light from the central part is interrupted. However, in Rheinberg illumination, the bright-field image becomes the background color for better discernment of the object ${ }^{17,18}$ and can simultaneously provide both bright-field and dark-field images using two-color light sources.

Figure 1 also presents various illumination patterns for multicontrast images with an LED array. Bright field, dark-field, and DPC images are obtained by changing the LED light source illumination pattern. ${ }^{12,13}$ A bright-field image is producible with 


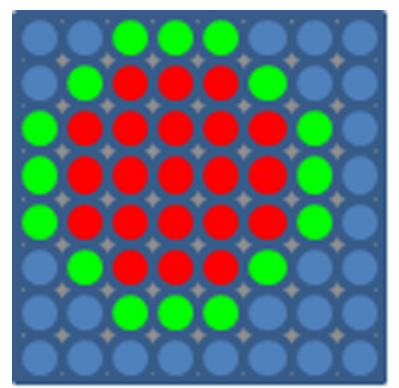

(a)

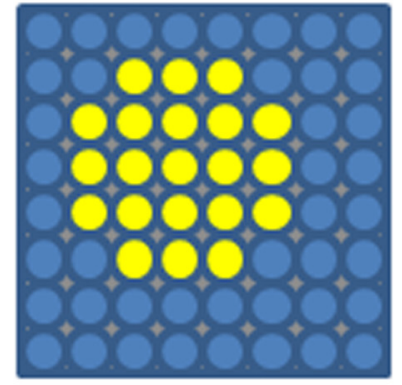

(b)

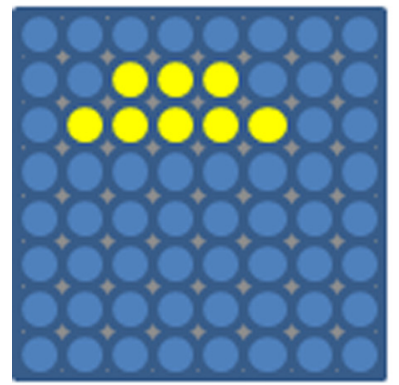

(e)

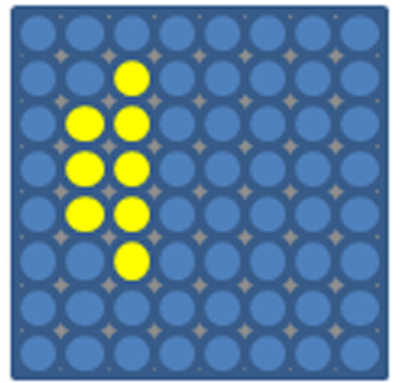

(c)

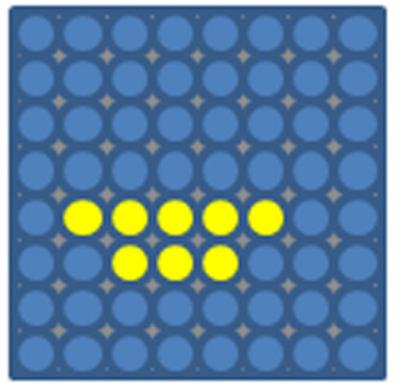

(f)

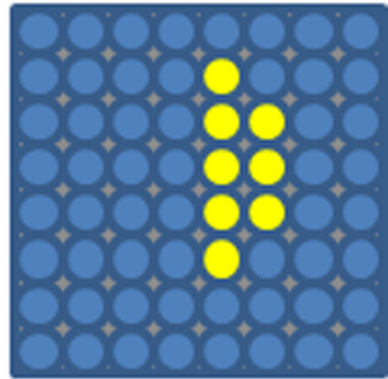

(d)

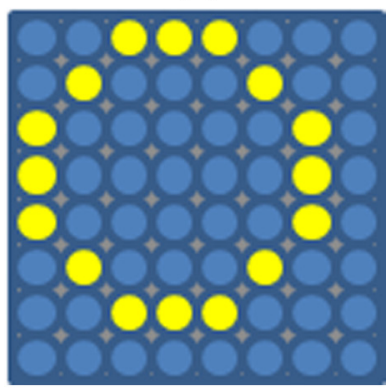

(g)

Fig. 1 Illumination patterns for multicontrast observation in the LED array used for this study: (a) a Rheinberg illumination pattern. Outer LED array (green LEDs) acts as dark-field illumination to visualize light-reflecting, refracting, diffusing, or scattering structures. The central LED array (red LEDs) acts as bright-field illumination to visualize light-absorbing structures; (b) bright-field pattern; (c) and (d) left-right DPC pattern; (e) and (f) top-bottom DPC pattern; and (g) dark-field pattern.

the illumination pattern displayed in Fig. 1(b). For bright-field images, the illumination angle is less than the NA of the objective. The phase contrast obtained by DPC derives from two asymmetric patterns of the illumination, as shown in Figs. 1(c) and 1(d). For example, images obtained with the left half of a circle and the right half of a circle are denoted, respectively, as $I_{\mathrm{L}}$ and $I_{\mathrm{R}}$. Then, the left-right DPC image $I_{\mathrm{LR} \_ \text {DPC }}$ is definable as follows: ${ }^{12,13}$

$I_{\mathrm{LR} \_\mathrm{DPC}}=\frac{I_{\mathrm{R}}-I_{\mathrm{L}}}{I_{\mathrm{R}}+I_{\mathrm{L}}}$.

Similarly, using the illumination in Figs. 1(e) and 1(f), the top-bottom DPC image $I_{\mathrm{TB} \_\mathrm{DPC}}$ is defined as follows:

$I_{\mathrm{TB} \_\mathrm{DPC}}=\frac{I_{\mathrm{T}}-I_{\mathrm{B}}}{I_{\mathrm{T}}+I_{\mathrm{B}}}$,

where $I_{\mathrm{T}}$ and $I_{\mathrm{B}}$, respectively, denote images obtained with the top and bottom halves of a circle illumination. An illumination scheme presented in Fig. 1(g) produces a dark-field image when the illumination angle is greater than the NA of the objective.
Consequently, in an LED array microscope, bright-field and dark-field images are obtained directly by changing the illumination patterns; DPC images are obtained with two asymmetric patterns of illumination and simple calculation but with no moving parts.

\section{Experimental Setup}

The schematic for a mobile-phone-based LED array microscope is presented in Fig. 2(a). The microscope consists of an objective lens, an eyepiece, and a smartphone camera. An objective lens (LMPlanFL 50×; Olympus Corp.) with an NA of 0.50 was used. An RGB full color dot matrix LED array $(8 \times 8$ dot, Product ID; 1487, Adafruit Industries) was used as a light source. Among 64 LED bulbs, we used 37 bulbs at maximum to obtain multimodal images as presented in Fig. 1. The eyepiece was used with magnification of 10 and field number of 22 (WHN10X; Olympus Corp.). We implemented the objective lens and eyepiece in a barrel (TV-IE; Chuo Precision Industrial Co. Ltd.). Light from the LED array illuminates the specimen. Light transmitted from the specimen was recorded as an image on the smartphone camera (resolution $3264 \times 2448$, iPhone5S; Apple Inc.). The $f$ number of the smartphone lens was 2.2. Images were stored in 


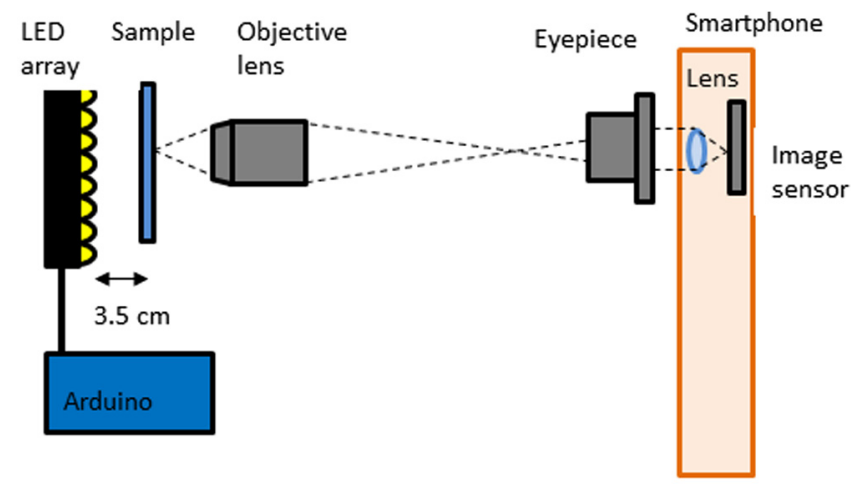

(a)

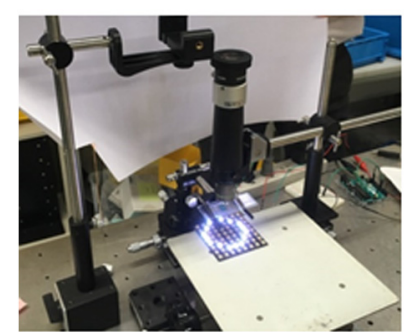

(b)

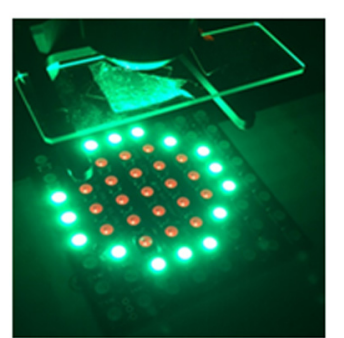

(c)
Fig. 2 (a) Schematic for the smartphone-based LED array microscope in this study. (b) Image of the fabricated smartphone-based LED array microscope. (c) Typical illumination pattern in the LED array to obtain a Rheinberg image.

TIFF format using application software: ProCamera. The LED array illumination pattern was controlled by an Arduino device. The distance between the sample and the LED array was set as $3.5 \mathrm{~cm}$. The distance between the smartphone and the eyepiece was $2.2 \mathrm{~cm}$. Figure 2(b) portrays a diagram of a constructed smartphone-based LED array microscope. Figure 2(c) depicts a typical illumination pattern for the LED array to obtain a Rheinberg image.

\section{Experimental Results}

\subsection{Observation of Living Moss Leaf Cells with Rheinberg Illumination}

Figures 3-6 present Rheinberg images of living leaf cells of moss, Physcomitrella patens (Physcomitrella). Mosses generally have extensive environmental and stress response capabilities. ${ }^{19}$ Because Physcomitrella is a model moss for molecular, cellular, and developmental biology, ${ }^{20,21}$ we chose Physcomitrella as the first observation target of our field-portable microscopy. In Fig. 3, the central red LED array acted as bright-field illumination. Outer green LEDs acted as dark-field illumination. By this arrangement, we changed the ratio between red LED intensity and green LED intensity. The intensities of the respective LEDs were controlled as values of 0 to 255 . The green intensity of the LED for dark-field illumination was set as the maximum of 255 . The red/green ratios portrayed in Figs. 3(a)-3(d) are, respectively, 255/255, 128/255, 64/255, and $32 / 255$. We obtained high-contrast images of the living cells. Visualization at the red/green ratio of 64/255 was easy to be identified. In the following experiments, we presented results at the intensity ratio of the inner circle/outer ring of 64/255. Figure 4 shows Rheinberg images of living Physcomitrella

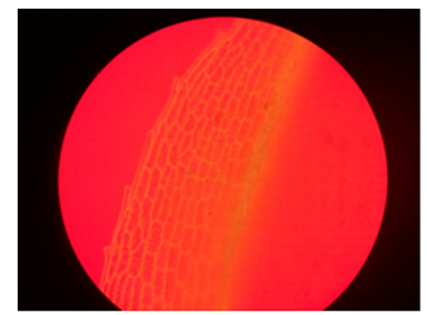

(a)

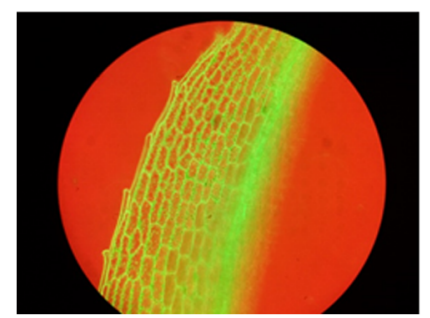

(c)

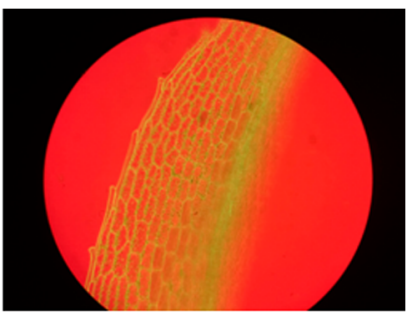

(b)

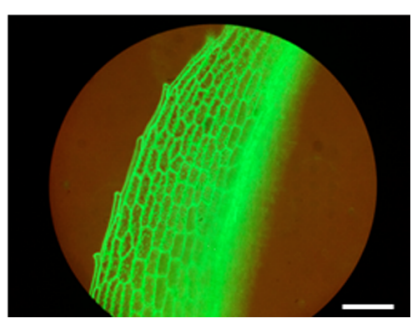

(d)
Fig. 3 Rheinberg images of living Physcomitrella leaf cells. The central red LED array acted as bright-field illumination. Outer green LEDs acted as dark-field illumination. The intensity of each LED was controlled to a value of 0 to 255. Red/green ratios: (a) 255/255, (b) 128/ 255, (c) 64/255, and (d) 32/255. Scale bar: $100 \mu \mathrm{m}$.

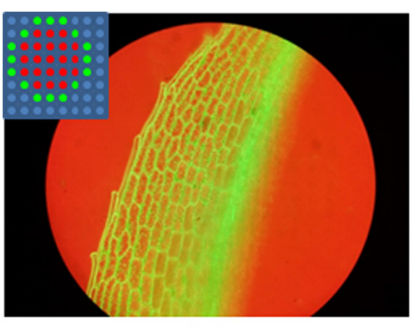

(a)

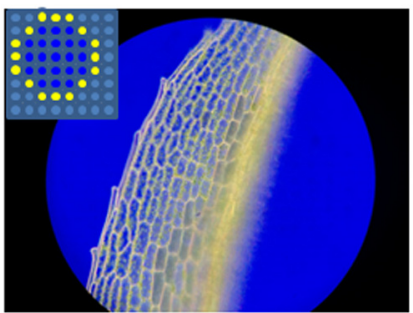

(c)

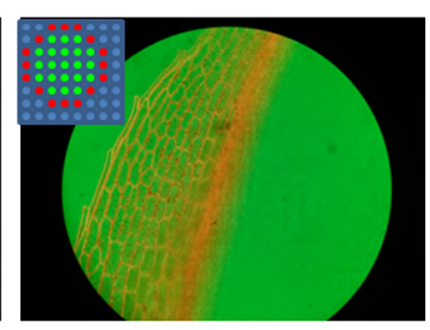

(b)

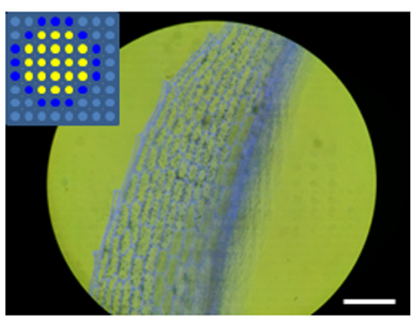

(d)
Fig. 4 Rheinberg images of living Physcomitrella leaf cells. Inner circle/outer ring: (a) red/green, (b) green/red, (c) blue/yellow, and (d) yellow/blue. The inner circle/outer ring intensity ratio was 64/ 255. Scale bar: $100 \mu \mathrm{m}$.

leaf cells with illumination combinations of various colors at the intensity ratio of the inner circle/outer ring of 64/255. Distinctive shapes of the cells can be visualized under any conditions. However, small differences were apparent among images obtained using various illumination combinations.

To analyze the differences further, we split colors of the Rheinberg images using ImageJ software (Fig. 5). Differences were not readily apparent between images obtained with red/ green illumination [Fig. 5(a)] and green/red illumination [Fig. 5(b)]. By contrast, images obtained with yellow/blue and blue/yellow illuminations mutually differed. Bright spots of chloroplasts with yellow outer illumination [Fig. 5(c), right 

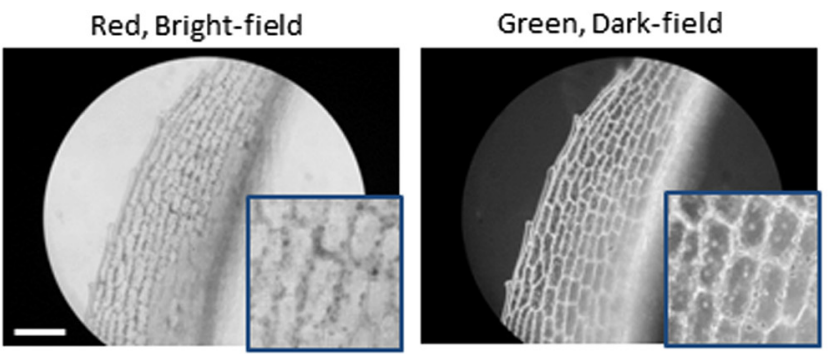

(a)
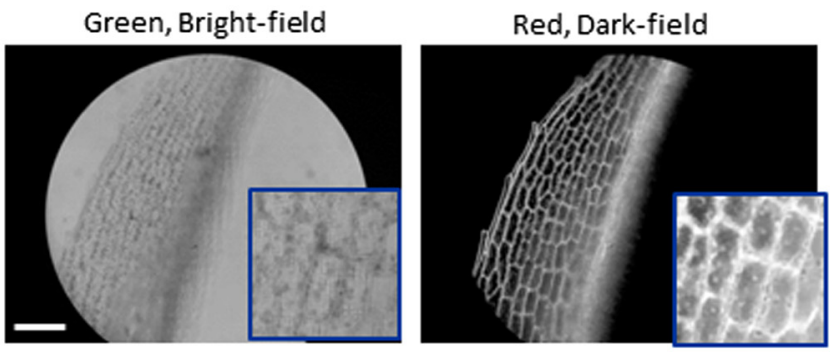

(b)
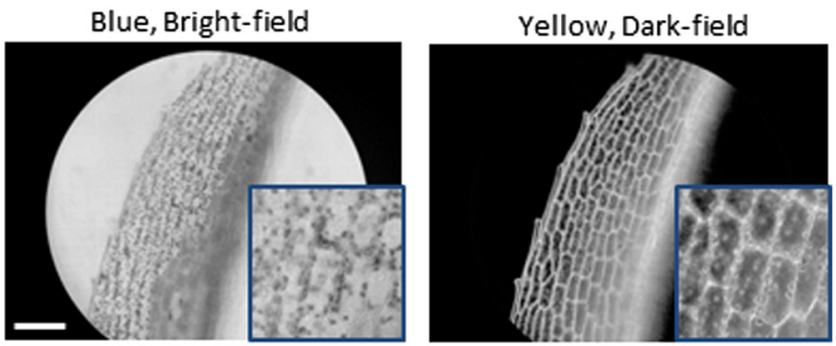

(c)
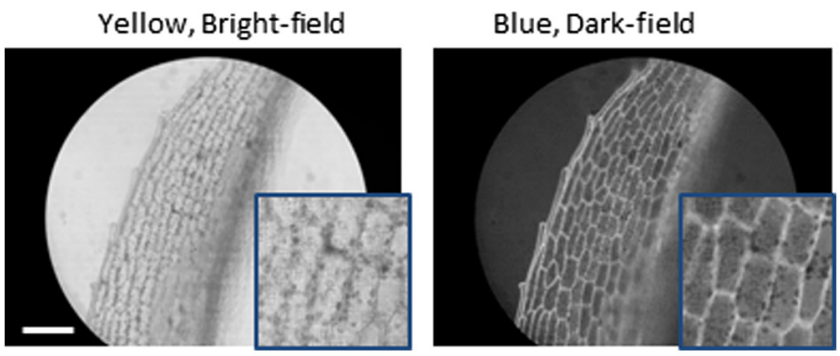

(d)

Fig. 5 Color splitting of Rheinberg images of living Physcomitrella leaf cells by image processing: (a) red inner illumination/green outer illumination, (b) green inner illumination/red outer illumination, (c) blue inner illumination/yellow outer illumination, and (d) yellow inner illumination/blue outer illumination. Yellow channels were produced by adding green and red channels. Scale bar: $100 \mu \mathrm{m}$.

panel] were similar to those with green and red outer ones. By contrast, chloroplasts appeared as dark spots with blue inner illumination [Fig. 5(c), left panel], which were darker than those with red, green, and yellow inner illuminations, leading to better contrast of chloroplasts in the Rheinberg images with blue inner/yellow outer illumination. However, with blue outer illumination, chloroplasts appeared as dark spots [Fig. 5(d), right panel], which differed from bright spots obtained with green, red, and yellow outer illuminations. Images with yellow inner illumination [Fig. 5(d), left panel] were similar to those with red and green inner ones. Taken together, in the Rheinberg images with a yellow inner/blue

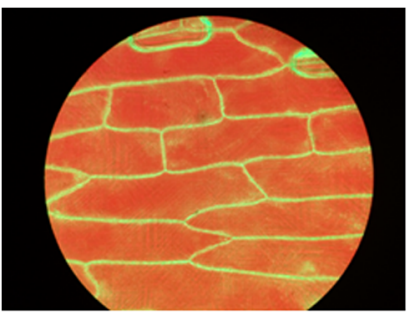

(a)

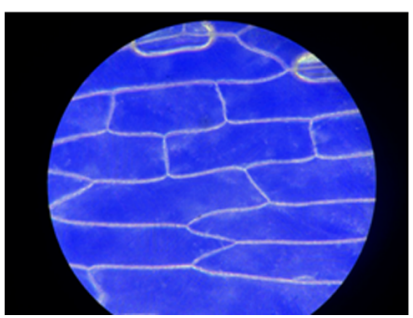

(c)

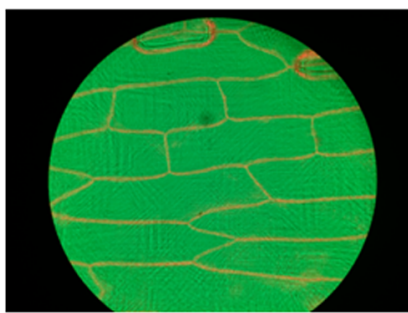

(b)

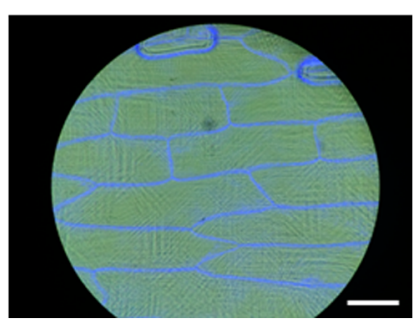

(d)
Fig. 6 Rheinberg images of living onion epidermal cells. Inner circle/ outer ring: (a) red/green, (b) green/red, (c) blue/yellow, and (d) yellow/ blue. The intensity ratio of inner circle/outer ring was 64/255. Scale bar: $100 \mu \mathrm{m}$.

outer illumination, the contrast at chloroplasts was diminished, leading to better visualization of other nonchromatic structures such as cell walls.

The differences described above are most likely caused by the chromatic character of chloroplasts: both chlorophylls and carotenoids, main pigments in chloroplasts, strongly absorb blue light. ${ }^{22-24}$ Therefore, chloroplasts appeared dark, even with dark-field blue light illumination [Fig. 5(d), right panel]. Chlorophylls (but not carotenoids) also absorb red light, although images with red light illumination are not distinguished from those with green and yellow light. This result might derive from green light absorbance by carotenoids. ${ }^{22-24}$

\subsection{Observation of Living Onion Epidermal Cells with Rheinberg Illumination}

Next, we observed living onion epidermal cells, in which no chloroplasts exist, with a mobile-phone-based Rheinberg microscope (Figs. 6 and 7). Figure 6 depicts the results with multiple combinations of Rheinberg illumination, of which the intensity ratio of inner circle/outer ring was 64/255. In all cases, cell shapes can be visualized. Figure 7 exhibits color splitting of Rheinberg images. With dark-field illuminations, cell walls were observed clearly.

\subsection{Bright-Field, Dark-Field, and Differential-Phase Contrast Imaging with RGB Illumination}

Figure 8 presents multicontrast images of living Physcomitrella leaf cells. Figures 8(a) and 8(b) show bright-field and dark-field images illuminated with RGB LEDs. Figures 8(c) and 8(d), respectively, portray left-right and top-bottom DPC images. In the dark-field image [Fig. 8(b)], cell wall and chloroplasts, respectively, show whitish and yellow-greenish colors, suggesting blue-light absorbance of chloroplasts. In DPC images, cell walls were clearly apparent along vertical and horizontal directions [Figs. 8(c) and 8(d)]. It is possible to emphasize the contrast of the cells in the same direction as the lighting 

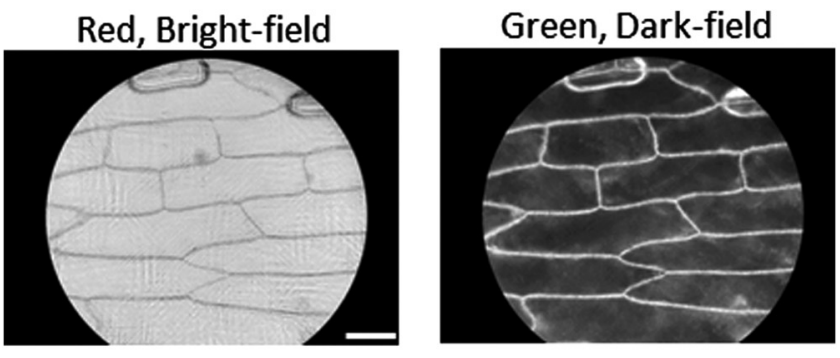

(a)
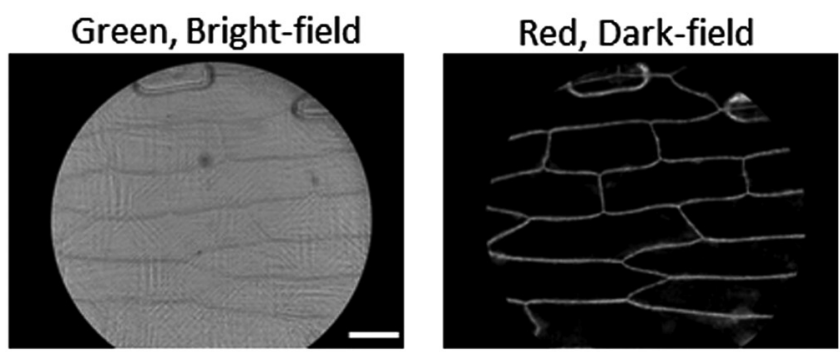

(b)
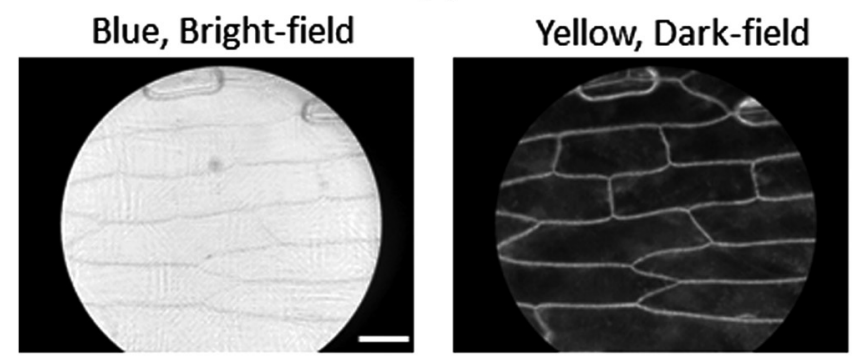

(c)
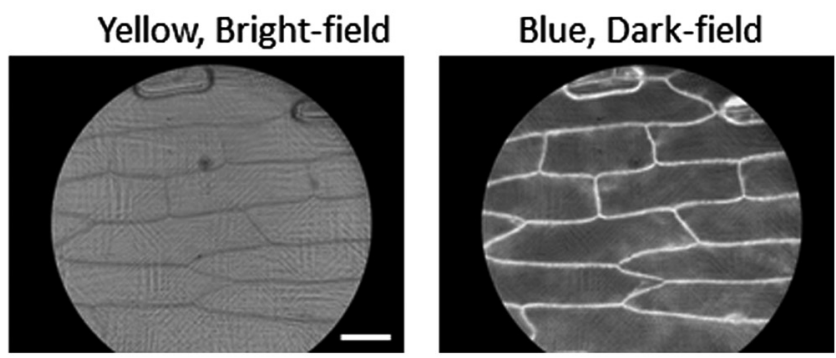

(d)

Fig. 7 Color splitting of Rheinberg images of living onion epidermal cells by image processing: (a) red inner illumination/green outer illumination, (b) green inner illumination/red outer illumination, (c) blue inner illumination/yellow outer illumination, and (d) yellow inner illumination/blue outer illumination. Yellow channels were produced by adding green and red channels. Scale bar: $100 \mu \mathrm{m}$.

pattern in the DPC because DPC imaging is based on the principle of oblique illumination.

Figure 9 shows multicontrast images of onion epidermal cells. Bright-field [Fig. 9(a)] and dark-field images [Fig. 9(b)] were obtained with the illumination of RGB LEDs. Figures 9(c) and 9(d), respectively, show left-right and top-bottom DPC images. Structural details along the DPC axis were enhanced in the DPC images.

\section{Discussion}

Living samples, a main observation target of examination using field portable microscopes, generally exhibit movement. In fact,

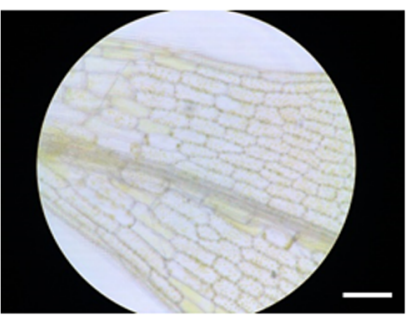

(a)

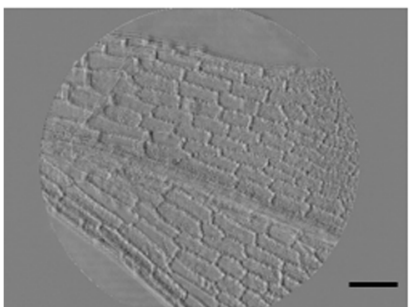

(c)

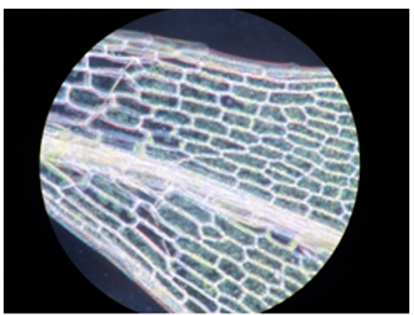

(b)

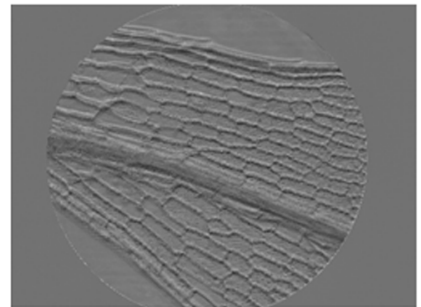

(d)
Fig. 8 Multicontrast images of living Physcomitrella leaf cells taken by controlled illumination patterns of the LED array: (a) bright-field, (b) dark-field, (c) left-right DPC, and (d) top-bottom DPC images. Scale bar: $100 \mu \mathrm{m}$.

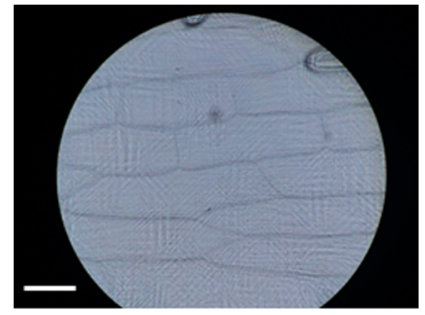

(a)

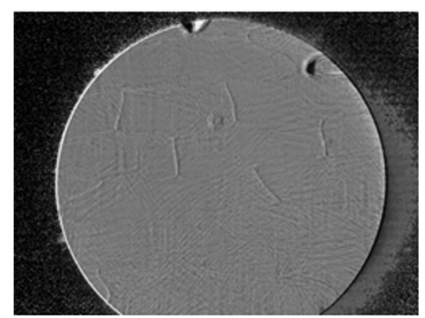

(c)

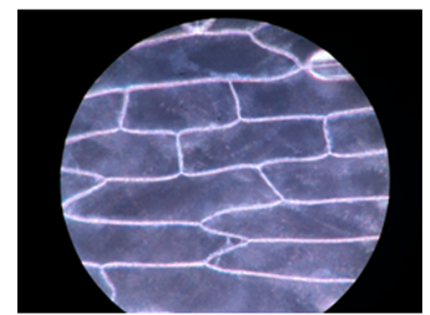

(b)

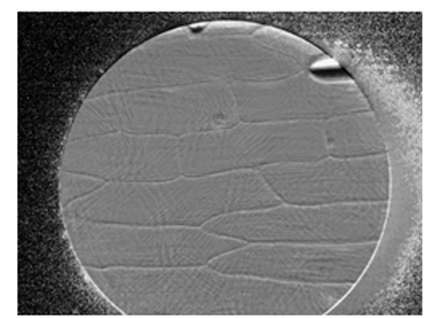

(d)
Fig. 9 Multicontrast images of onion epidermal cells under (a) brightfield and (b) dark-field illumination. DPC images of (c) left-right and (d) top-bottom DPCs. Scale bar: $100 \mu \mathrm{m}$.

not only micro-organisms and animals, but also even sessile plant cells show movement. Subcellular structures actively move, exhibiting motion, such as protoplasmic flow. Therefore, a field portable microscope is expected to be useful for simultaneous acquisition of bright-field and dark-field images in Rheinberg illumination. Using various color combinations of Rheinberg illumination, the contrast at chromatic structures, such as chloroplasts, can be enhanced or diminished. In addition, chromatic characterization is possible with Rheinberg illumination. We found a certain structure that disturbs blue and green light but not yellow and red light, in onion epidermal cells. In the color-code LED array, ${ }^{15}$ a central bright region is divided to regions of two colors. When a sample has absorption, 
the color-code LED array is not easy to use. In Rheinberg illumination, bright-field illumination uses only one color. Therefore, light-absorbing structures can be visualized.

The field-of-view of the constructed microscope was measured as $\sim 650 \mu \mathrm{m}$. Through sample observation, aberration was confirmed. Because of field curvature, the image is blurred as it goes to the edge. Pincushion type distortion was also observable. This aberration is probably attributable to the smartphone lens. The aberration effect shown by a mobile phone camera can be corrected using image processing. ${ }^{15}$ The resolution of the constructed smartphone microscope was measured. We used a 1951 USAF test target (\#38257; Edmond Optics). From the bright-filed images with RGB illumination, the contrast transfer function was higher than 0.74 . We also measured the 1951 USAF test target with Rheinberg illumination (red inner illumination/green outer illumination, green inner illumination/red outer illumination, blue inner illumination/yellow outer illumination, and yellow inner illumination/blue outer illumination). It is possible to visualize a test target of 228 lines pair $/ \mathrm{mm}$ with group 7 and element 6 . Consequently, the resolution was calculated as less than $4.4 \mu \mathrm{m}$.

\section{Conclusion}

For this study, observations of living cells were conducted using a smartphone microscope with an $8 \times 8$ LED array. Using only 37 LED bulbs, we demonstrated multimodal imaging with bright-field, dark-field, DPC, and Rheinberg illuminations. Rheinberg illumination enables simultaneous acquisition of both bright-field and dark-field images using two-color light sources. By changing the color combinations, the chromatic structure can be visualized with higher or lower contrast. Chromatic characterization is also possible. The multimodal smartphone-based LED array microscope is promising for potential application to cellular and subcellular observations conducted in the field.

\section{Disclosures}

The authors have no relevant financial interests in this article and no potential conflicts of interest to disclose.

\section{Acknowledgments}

The authors thank Kazuko Koda and Shuu Uenoyama of Ritsumeikan University for setting up the optical system. W.W. acknowledges JSPS KAKENHI Grant-in-Aid for Scientific Research (C) under Grant No. 26390088.

\section{References}

1. A. J. Nagano et al., "Deciphering and prediction of transcriptome dynamics under fluctuating field conditions," Cell 151(6), 1358-1369 (2012).

2. K. S. Araki, T. Kubo, and H. Kudoh, "Genet-specific DNA methylation probabilities detected in a spatial epigenetic analysis of a clonal plant population," PLoS One 12(5), e0178145 (2017).

3. Z. J. Smith et al., "Cell-phone-based platform for biomedical device development and education applications," PLoS One 6(3), e17150 (2011).

4. A. Arpa et al., "Single lens off-chip cellphone microscopy," in IEEE Computer Society Conf. on Computer Vision and Pattern Recognition Workshops (CVPRW) (2012).

5. A. R. Miller et al., "Portable, battery-operated, low-cost, bright field and fluorescence microscope," PLoS One 5(8), e11890 (2010).

6. X. Meng, H. Huang, and K. Yan, "Smartphone based hand-held quantitative phase microscope using the transport of intensity equation method," Lab Chip 17, 104-109 (2017).
7. D. N. Breslauer et al., "Mobile phone based clinical microscopy for global health applications," PLoS One 4(7), e6320 (2009).

8. A. Skandarajah et al., "Quantitative imaging with a mobile phone microscope," PLoS One 9(5), e96906 (2014).

9. C. W. Pirnstill and G. L. Coté, "Malaria diagnosis using a mobile phone polarized microscope," Sci. Rep. 5, 13368 (2015).

10. A. Orth et al., "A dual-mode mobile phone microscope using the onboard camera flash and ambient light," Sci. Rep. 8, 3298 (2018).

11. G. Zheng, C. Kolner, and C. Yang, "Microscopy refocusing and darkfield imaging by using a simple LED array," Opt. Lett. 36(20), 39873989 (2011).

12. L. Tian, J. Wang, and L. Waller, "3D differential phase-contrast microscopy with computational illumination using an LED array," Opt. Lett. 39(5), 1326-1329 (2014).

13. Z. Liu et al., "Real-time brightfield, darkfield, and phase contrast imaging in a light-emitting diode array microscope," J. Biomed. Opt. 19(10), 106002 (2014).

14. Z. F. Phillips et al., "Multi-contrast imaging and digital refocusing on a mobile microscope with a domed LED array," PLoS One 10(5), e0124938 (2015).

15. D. Jung et al., "Smartphone-based multi-contrast microscope using color-multiplexed illumination," Sci. Rep. 7, 7564 (2017).

16. K. Koda et al., "Image acquisition with smartphone-based LED array microscope," Proc. SPIE 10711, $107111 \mathrm{Z}$ (2018).

17. M. W. Davidson and M. Abramowitz, "Optical microscopy," Encycl. Imagning Sci. Technol. 2, 1106-1140 (2002).

18. E. C. Samson and C. M. Blanca, "Dynamic contrast enhancement in widefield microscopy using projector-generated illumination patterns," New. J. Phys. 9, 363-363 (2007).

19. A. C. Cuming, "Moss as a model system for plant stress responses," in Plant Stress Biology: From Genomics to Systems Biology, H. Hirt, Ed., pp. 17-36, Wiley-VCH Verlag (2010).

20. S. A. Rensing et al., "The Physcomitrella genome reveals evolutionary insights into the conquest of land by plants," Science 319, 64-69 (2008)

21. R. Kofuji and M. Hasebe, "Eight types of stem cells in the life cycle of the moss Physcomitrella patens," Curr. Opin. Plant Biol. 17, 13-21 (2014).

22. G. S. Rabideau, C. S. French, and A. S. Holt, "The absorption and reflection spectra of leaves, chloroplast suspensions, and chloroplast fragments as measured in an Ulbricht sphere," Am. J. Bot. 33, 769-777 (1946).

23. H. K. Lichtenthaler, "Chlorophylls and carotenoids: pigments of photosynthetic biomembranes," Methods Enzymol. 148, 350-382 (1987).

24. R. R. Bidigare et al., "In-vivo absorption properties of algal pigments," Proc. SPIE 1302, 290-302 (1990).

Hidenobu Arimoto received his $\mathrm{PhD}$ in engineering from Osaka University, Osaka, Japan, in 2000. He is now a researcher of the National Institute of Advanced Industrial Science and Technology, Tsukuba, Japan. His research interests include biomedical optics and optical signal processing.

Yosuke Tamada is an assistant professor at the National Institute for Basic Biology in Japan. He received his BS degree in agriculture in 2000, and his MS and PhD degrees in life science in 2002 and 2005, respectively, from Kyoto University. His current research interests include developmental biology and live-cell imaging using plants. $\mathrm{He}$ is a member of the Optical Society of Japan.

Wataru Watanabe is a professor at Ritsumeikan University. He received his $\mathrm{BS}, \mathrm{MS}$, and doctor of engineering degrees from Osaka University in 1994, 1996, and 1999, respectively. During 1999-2006, he was an assistant professor at the Graduate School of Engineering, Osaka University. During 2006 to 2013, he was a researcher at the National Institute of Advanced Industrial Science and Technology (AIST). He joined Ritsumeikan University in 2013 as a professor. $\mathrm{He}$ is the author of more than 50 journal papers. His current research interests include ultrafast laser micromachining and biomedical optics. He is a member of SPIE.

Biographies for the other authors are not available. 